\title{
Capsule Commentary on Duong et al., Exploring Physician Perspectives of Residency Holdover Handoffs: a Qualitative Study to Understand an Increasingly Important Type of Handoff
}

\author{
Gregory M. Bump, MD, FACP \\ Division of General Internal Medicine, University of Pittsburgh Medical Center, Pittsburgh, PA, USA.
}

J Gen Intern Med 32(6):682

DOI: $10.1007 / \mathrm{s} 11606-017-4016-\mathrm{Z}$

(c) Society of General Internal Medicine 2017

$\mathrm{H}$ old-over admissions are common during internal medicine training. A hold-over patient is one admitted in the evening hours by a night team who transfers patient care responsibility to a day team the next morning. While multicenter studies are lacking, single-center studies suggest that $40-45 \%$ of residency general medicine admissions are hold-overs. ${ }^{1,2}$ Discontinuity of care, when patients are admitted by one team and handed off to another team, is error prone. Despite the regularity of hold-over admissions, very little is known about how to approach them differently than other hand-offs. What can we do to make them safe? What can we do to make them educational? Duong et al. ${ }^{1}$ offer practical answers.

The authors interviewed resident physicians and hospitalists. Several straightforward strategies emerged. Hand-offs should be performed with immediate access to the medical record to verify information that requires interpretation (for example, an EKG). The authors highlight the utility of focusing the hand-off less on facts and more on clinical decision making. Being explicit about diagnostic uncertainty is helpful. When the admitting team is less confident in their diagnosis, the day team can adjust their history and examination to ensure they agree (or disagree) with the admitting diagnosis. Such statements are more likely to be made verbally than written. Therefore hand-offs should be face-to-face with an opportunity to ask questions. The authors also discuss the benefits to the admitting team of getting feedback about how the patient did.
While this work offers practical advice to improve the safety and educational value of hold-overs, an important question exists for medical educators. Two influential studies examined relaxed duty-hour restrictions in surgery $^{3}$ and medicine. ${ }^{4}$ The results in medicine are not yet published. Recently, the ACGME proposed relaxed duty hour rules, which allow interns to have overnight call. ${ }^{5}$ The question for educators could be whether to implement strategies to improve hold-overs or to restructure their admitting services to decrease discontinuity. Are hold-overs here to stay and should we improve them, or should we make them less common?

Corresponding Author: Gregory M. Bump, MD, FACP; Division of General Internal MedicineUniversity of Pittsburgh Medical Center, Pittsburgh, PA, USA (e-mail: bumpgm@upmc.edu).

\section{Compliance with Ethical Standards:}

Conflict of Interest: The author has no conflicts of interest with this article.

\section{REFERENCES}

1. Duong JA, Jensen TP, Morduchowicz S, Mourad M, Harrison JD, Ranji SR. Exploring physician perspectives of residency holdover handoffs: a qualitative study to understand an increasingly important type of handoff. J Gen Intern Med. doi: 10.1007/s11606-017-4009-y

2. Bump GM, Zimmer SM, McNeil MA, Elnicki DM. Hold-over admissions: are they educational for residents? J Gen Intern Med. 2013;29(3):467-7.

3. Bilimoria KY, Chung JW, Hedges LV, et al. National cluster-randomized trial of duty-hour flexibility in surgical training. N Engl $\mathrm{J}$ Med. 2016;374:713-727.

4. Individualized Comparative Effectiveness of Models Optimizing Patient Safety and Resident Education. (iCOMPARE). Accessed at: http://www.jhcct.org/icompare/default.asp on February 8, 2017.

5. ACGME Common Program Requirements Proposed Major Revisions. Accessed at: http://www.acgme.org/Portals/O/PFAssets/ ReviewandComment/CPR_SectionVI_ChangesTracked.pdf on February 8, 2017.

Published online February 27, 2017 